\title{
Non-uniform Bound on Normal Approximation of Latin Hypercube Sampling
}

\author{
Kritsana Neammanee \\ Department of Mathematics, Faculty of Science \\ Chulalongkorn University \\ Bangkok 10330, Thailand \\ E-mail: Kritsana.N@chula.ac.th \\ Petcharat Rattanawong \\ Faculty of Science and Social Science \\ Burapha University Sakaew Campus \\ Sakeaw 27160, Thailand \\ E-mail: petchar@buu.ac.th
}

\begin{abstract}
Latin hypercube sampling(LHS)(McKay, M.D., 1979) is a method of sampling that can be used to estimate the value of multidimensional integration. Loh(Loh, W.L., 1996b) and Neammanee and Rattanawong(Neammanee, K., 2009) gave a uniform bound in normal approximation for LHS. In this paper, we give a non-uniform bound of this approximation by using Stein's method.
\end{abstract}

Keywords: Non-uniform bound, Latin hypercube sampling, Stein's method

\section{Introduction and Main Results}

Latin hypercube sampling(McKay, M.D., 1979) is a method of sampling that can be used to produce input values for estimation of integrals over multidimensional domains. The main feature of Latin hypercube sampling(LHS) is that, contrast to simple random sampling, it stratifies on all input dimensions simultaneously. More precisely, for positive integers $d$ and $n, d \geq 2$, let:

1. $\pi_{k}, 1 \leq k \leq d$, be random permutations of $\{1, \ldots, n\}$ each uniformly distributed over all the $n$ ! possible permutations;

2. $U_{i_{1}, \ldots, i_{d}, j} 1 \leq i_{1}, \ldots, i_{d} \leq n, 1 \leq j \leq d$, be $[0,1]$ uniform random variables;

3. The $U_{i_{1}, \ldots, i_{d}, j}$ 's and $\pi_{k}$ 's all be stochastically independent.

A Latin hypercube sample of size $n$ (taken from the $d$-dimensional hypercube $\left.[0,1]^{d}\right)$ is defined to be $\left\{X\left(\pi_{1}(i), \pi_{2}(i), \ldots, \pi_{d}(i)\right)\right.$ : $1 \leq i \leq n\}$, where for all $1 \leq i_{1}, \ldots, i_{d} \leq n$,

$$
\begin{aligned}
X_{j}\left(i_{1}, \ldots, i_{d}\right) & =\left(i_{j}-U_{i_{1}, \ldots, i_{d}, j}\right) / n \quad \forall 1 \leq j \leq d, \\
X\left(i_{1}, \ldots, i_{d}\right) & =\left(X_{1}\left(i_{1}, \ldots, i_{d}\right), \ldots, X_{d}\left(i_{1}, \ldots, i_{d}\right)\right) .
\end{aligned}
$$

Let $X$ be a random vector uniformly distributed on $[0,1]^{d}$ and $f$ be a measurable function from $[0,1]^{d}$ to $\mathbb{R}$. In many problem, we would like to estimate $\mu=\int_{[0,1]^{d}} f(x) d x$. We note that $E(f \circ X)=\int_{[0,1]^{d}} f(x) d x$. The estimator for $\mu$ that based on a Latin hypercube sampling is

$$
\hat{\mu}_{n}=\frac{1}{n} \sum_{k=1}^{n} f \circ X\left(\pi_{1}(k), \pi_{2}(k), \ldots, \pi_{d}(k)\right) .
$$

Then $\hat{\mu}_{n}$ is an unbiased estimator for $\mu$. McKay Beckman and Conover(McKay, M.D., 1979) showed that in a great number of instances, the varaince of $\hat{\mu}_{n}$ is substantially smaller than that of the estimator based on simple random sampling. Stein(Stein, M.L., 1987) further proved that the asymptotic variance of $\hat{\mu}_{n}$ is less than the asymtotic variance of an 
analogous estimator based on an independently and identically distributed sample. Owen(Owen,A.B., 1992) showed that the multivariate central limit theorem holds for $\hat{\mu}_{n}$ when $f$ is a bounded function. Assume that $\operatorname{Var}\left(\hat{\mu}_{n}\right)>0$, we define

$$
W=\frac{\hat{\mu}_{n}-\mu}{\sqrt{\operatorname{Var}\left(\hat{\mu}_{n}\right)}} .
$$

In 1996, Loh(Loh, W.L., 1996) gave a uniform bound on the normal approximation of $W$ but he yield the convergence rate $\frac{C}{\sqrt{n}}$ under the finiteness of third moments without the value of $C$. Neammanee and Rattanawong(Neammanee, K., 2009) give a constant $C$ by using Stein's method. Their result described in Theorem 1.1.

Theorem 1.1 For each $i_{1}, \ldots, i_{d} \in\{1, \ldots, n\}$, let

$$
\begin{aligned}
\mu\left(i_{1}, \ldots, i_{d}\right) & =E f \circ X\left(i_{1}, \ldots, i_{d}\right), \\
U_{k}\left(i_{1}, \ldots, i_{d}\right) & =\frac{(-1)^{k}}{n^{k}} \sum_{1 \leq j_{1}<j_{2}<\ldots<j_{k} \leq d} \sum_{q_{j_{1}}=1}^{n} \ldots \sum_{q_{j_{k}}=1}^{n} \mu\left(l_{1}, \ldots, l_{d}\right),
\end{aligned}
$$

where

$$
l_{p}= \begin{cases}q_{p} & \text { if } p=j_{1}, \ldots, j_{k} \\ i_{p} & \text { otherwise }\end{cases}
$$

and

$$
Y\left(i_{1}, \ldots, i_{d}\right)=\frac{1}{n \sqrt{\operatorname{var}\left(\hat{\mu}_{n}\right)}}\left[f \circ X\left(i_{1}, \ldots, i_{d}\right)+\sum_{k=1}^{d-1} U_{k}\left(i_{1}, \ldots, i_{d}\right)+(-1)^{d} \mu\right]
$$

Suppose that $E\left(f \circ X\left(i_{1}, \ldots, i_{d}\right)\right)^{4}<\infty, 1 \leq i_{1}, \ldots, i_{d} \leq n$. Then for $n \geq 6^{d}+3$,

$$
\begin{aligned}
\sup _{z \in \mathbb{R}}|P(W \leq z)-\Phi(z)| \leq & \frac{\sqrt{d} \delta_{4}^{\frac{1}{4}}}{n^{\frac{3}{8}}}+\frac{11.68}{\sqrt{n}}+8.845 d^{\frac{1}{4}} \delta_{4}+12.513 \delta_{4}+\frac{5.014 d \delta_{4}^{\frac{3}{4}}}{n^{\frac{1}{8}}} \\
& +\frac{2 \sqrt{2 \pi} \delta_{4}^{\frac{3}{4}}}{n^{\frac{1}{8}}}
\end{aligned}
$$

where $\Phi$ is the standard normal distribution, and

$$
\delta_{k}=\frac{1}{n^{d-\frac{k-1}{2}}} \sum_{i_{1}=1}^{n} \ldots \sum_{i_{d}=1}^{n} E\left|Y\left(i_{1}, \ldots, i_{d}\right)\right|^{k}
$$

In this paper, we give a non-uniform bound on the normal approximation of $W$. Here is our result.

Theorem 1.2 Suppose $E\left(f \circ X\left(i_{1}, \ldots, i_{d}\right)\right)^{6}<\infty$. Then for $z \in \mathbb{R}$,

$$
|P(W \leq z)-\Phi(z)| \leq \frac{C}{1+|z|}\left\{\frac{\delta_{4}^{\frac{1}{4}}}{n^{\frac{3}{8}}}+n^{\frac{5}{4}} \delta_{4}^{\frac{7}{2}}+\delta_{3}+\delta_{6}^{\frac{1}{2}}\left(\delta_{4}^{\frac{1}{2}}+\delta_{4}\right)\right\} .
$$

Furthermore, if $\delta_{k} \sim n^{-1 / 2}(k=3,4,6)$,

$$
|P(W \leq z)-\Phi(z)| \leq \frac{C}{(1+|z|) \sqrt{n}}
$$

To obtain a non-uniform bound for orthogonal array, Laipaporn and Neammanee(Laipaporn, K., 2007) assume 30 $0^{\text {th }}$ moment. But in Theorem 1.2, we assume $6^{\text {th }}$ moment which is better than their result.

This paper is organized as follows. In section 2, we give a number of lemmas that are needed in proving our main theorem(Theorem 1.2). A proof of Theorem 1.2 is given in section 3 .

\section{Auxiliary Results}

In this section, we shall give auxiliary results for proving our main theorem.

Neammanee and Rattanawong (Neammanee, K., 2009) showed that there exist random permutations $\rho_{1}, \ldots, \rho_{d-1}$ on $\{1, \ldots, n\}$ such that 


$$
W=\sum_{i=1}^{n} Y\left(i, \rho_{1}(i), \ldots, \rho_{d-1}(i)\right)
$$

For fixed $z \geq 0$, we also define

$$
\begin{aligned}
& Y_{z}\left(i_{1}, \ldots, i_{d}\right)=Y\left(i_{1}, \ldots, i_{d}\right) \mathbb{I}\left(\left|Y\left(i_{1}, \ldots, i_{d}\right)\right|>1+z\right), \\
& \widehat{Y}_{z}\left(i_{1}, \ldots, i_{d}\right)=Y\left(i_{1}, \ldots, i_{d}\right) \mathbb{I}\left(\left|Y\left(i_{1}, \ldots, i_{d}\right)\right| \leq 1+z\right),
\end{aligned}
$$

wherellis the indicator function and

$$
\widehat{Y}(\rho)=\sum_{i=1}^{n} \widehat{Y}_{z}\left(i, \rho_{1}(i), \ldots, \rho_{d-1}(i)\right) .
$$

To define a coupling pair $\widetilde{Y}(\rho)$ of $\widehat{Y}(\rho)$, let $I$ and $K$ be uniformly distributed random variables on $\{1, \ldots, n\},(I, K)$ uniformly distributed on $\{(i, k) \mid i, k=1, \ldots, n, i \neq k\}$ and assume that they are independent of $\rho_{1}, \ldots, \rho_{d-1}$ and $Y\left(i_{1}, \ldots, i_{d}\right)$ 's.

Let

$$
\widetilde{Y}(\rho)=\widehat{Y}(\rho)-\widehat{S}_{1, z}-\widehat{S}_{2, z}+\widehat{S}_{3, z}+\widehat{S}_{4, z}
$$

where

$$
\begin{aligned}
& \widehat{S}_{1, z}=\widehat{Y}_{z}\left(I, \rho_{1}(I), \ldots, \rho_{d-1}(I)\right), \widehat{S}_{2, z}=\widehat{Y}_{z}\left(K, \rho_{1}(K), \ldots, \rho_{d-1}(K)\right), \\
& \widehat{S}_{3, z}=\widehat{Y}_{z}\left(I, \rho_{1}(K), . ., \rho_{d-1}(K)\right), \widehat{S}_{4, z}=\widehat{Y}_{z}\left(K, \rho_{1}(I), \ldots, \rho_{d-1}(I)\right)
\end{aligned}
$$

Note that, by the same argument as in Neammanee and Suntornchost(Neammanee, K., 2005), $\widehat{Y}(\rho)$ and $\widetilde{Y}(\rho)$ is an exchangeable pair and $\widehat{S}_{i, z}$ for $i=1,2,3,4$ are identically distributed.

Lemma 2.1 Let $g$ be a continuous and piecewise continuously differential function. Then,

$$
E \widehat{Y}(\rho) g(\widehat{Y}(\rho))=E \int_{-\infty}^{\infty} g^{\prime}(\widehat{Y}(\rho)+t) K(t) d t+\Delta g(\widehat{Y}(\rho))
$$

and

$$
|\Delta g(\widehat{Y}(\rho))| \leq C\left(\frac{\delta_{4}^{\frac{1}{4}}}{n^{\frac{3}{8}}}+\delta_{4}\right)\left(\operatorname{Eg}^{2}(\widehat{Y}(\rho))\right)^{\frac{1}{2}}
$$

where

$$
K(t)=\frac{n-1}{4}(\widetilde{Y}(\rho)-\widehat{Y}(\rho))(\mathbb{I}(0 \leq t \leq \widetilde{Y}(\rho)-\widehat{Y}(\rho))-\mathbb{I}(\widetilde{Y}(\rho)-\widehat{Y}(\rho) \leq t<0)) .
$$

proof Let $\mathcal{A}$ be the $\sigma$-algebra generated by

$$
\left\{\widehat{Y}_{z}\left(i, \rho_{1}(i), \ldots, \rho_{d-1}(i)\right): 1 \leq i \leq n\right\} .
$$

By the same argument as in Neammanee and Suntornchost(Neammanee, K., 2005), we can show that

$$
2 E\left\{g(\widehat{Y}(\rho)) E^{\mathcal{A}}(\widetilde{Y}(\rho)-\widehat{Y}(\rho))\right\}+E(\widetilde{Y}(\rho)-\widehat{Y}(\rho))[g(\widetilde{Y})(\rho)-g(\widehat{Y}(\rho))]=0 .
$$

From this fact and the fact that

$$
\begin{aligned}
E^{\mathcal{A}} & {[\widetilde{Y}(\rho)-\widehat{Y}(\rho)] } \\
= & E^{\mathcal{A}}\left[-\widehat{S}_{1, z}-\widehat{S}_{2, z}+\widehat{S}_{3, z}+\widehat{S}_{4, z}\right] \\
= & -E^{\mathcal{A}} \widehat{Y}_{z}\left(I, \rho_{1}(I), \ldots, \rho_{d-1}(I)\right)-E^{\mathcal{A}} \widehat{Y}_{z}\left(K, \rho_{1}(K), \ldots, \rho_{d-1}(K)\right) \\
& +E^{\mathcal{A}} \widehat{Y}_{z}\left(I, \rho_{1}(K), \ldots, \rho_{d-1}(K)\right)+E^{\mathcal{A}} \widehat{Y}_{z}\left(K, \rho_{1}(I), \ldots, \rho_{d-1}(I)\right) \\
= & -\frac{2}{n} \sum_{i=1}^{n} \widehat{Y}_{z}\left(i, \rho_{1}(i), \ldots, \rho_{d-1}(i)\right)+\frac{2}{n(n-1)} E^{\mathcal{A}} \sum_{i=1}^{n} \sum_{k} \widehat{Y}_{z}\left(i, \rho_{1}(k), \ldots, \rho_{d-1}(k)\right) \\
= & -\frac{2}{n} \widehat{Y}(\rho)+\frac{2}{n(n-1)} E^{\mathcal{A}} \sum_{i=1}^{n}\left\{\sum_{k=1}^{n} \widehat{Y}_{z}\left(i, \rho_{1}(k), \ldots, \rho_{d-1}(k)\right)\right. \\
& \left.-\widehat{Y}_{z}\left(i, \rho_{1}(i), \ldots, \rho_{d-1}(i)\right)\right\} \\
= & -\frac{2}{n-1} \widehat{Y}(\rho)+\frac{2}{n(n-1)} E^{\mathcal{A}} \sum_{i=1}^{n} \sum_{k=1}^{n} \widehat{Y}_{z}\left(i, \rho_{1}(k), \ldots, \rho_{d-1}(k)\right),
\end{aligned}
$$


we have

$$
\begin{aligned}
E \widehat{Y}(\rho) g(\widehat{Y}(\rho))= & \frac{n-1}{4} E(\widetilde{Y}(\rho)-\widehat{Y}(\rho))[g(\widetilde{Y}(\rho))-g(\widehat{Y}(\rho))] \\
& +\frac{1}{n} E g(\widehat{Y}(\rho)) \sum_{i=1}^{n} \sum_{k=1}^{n} \widehat{Y}_{z}\left(i, \rho_{1}(k), \ldots, \rho_{d-1}(k)\right) \\
= & \frac{n-1}{4} E(\widetilde{Y}(\rho)-\widehat{Y}(\rho))[g(\widetilde{Y}(\rho))-g(\widehat{Y}(\rho))]+\Delta g(\widehat{Y}(\rho)) \\
= & \frac{n-1}{4} E(\widetilde{Y}(\rho)-\widehat{Y}(\rho)) \int_{0}^{\widetilde{Y}(\rho)-\widehat{Y}(\rho)} g^{\prime}(\widehat{Y}(\rho)+t) d t+\Delta g(\widehat{Y}(\rho)) \\
= & E \int_{-\infty}^{\infty} g^{\prime}(\widehat{Y}(\rho)+t) K(t) d t+\Delta g(\widehat{Y}(\rho))
\end{aligned}
$$

where

$$
\begin{aligned}
|\Delta g(\widehat{Y}(\rho))| & =\frac{1}{n} E\left|g(\widehat{Y}(\rho)) \sum_{i=1}^{n} \sum_{k=1}^{n} \widehat{Y}_{z}\left(i, \rho_{1}(k), \ldots, \rho_{d-1}(k)\right)\right| \\
& \leq \frac{1}{n}\left\{E g^{2}(\widehat{Y}(\rho))\right\}^{\frac{1}{2}}\left\{E\left[\sum_{i=1}^{n} \sum_{k=1}^{n} \widehat{Y}_{z}\left(i, \rho_{1}(k), \ldots, \rho_{d-1}(k)\right)\right]^{2}\right\}^{\frac{1}{2}} .
\end{aligned}
$$

The proof will be completed, if we can show that

$$
E\left[\sum_{i=1}^{n} \sum_{k=1}^{n} \widehat{Y}_{z}\left(i, \rho_{1}(k), \ldots, \rho_{d-1}(k)\right)\right]^{2} \leq C\left(n^{\frac{5}{4}} \delta_{4}^{\frac{1}{2}}+n^{2} \delta_{4}^{2}\right) .
$$

By the fact that

$$
\sum_{i_{j}}^{n} E Y\left(i_{1}, \ldots, i_{d}\right)=0
$$

for each $j \in\{1, \ldots, d\}$ (Neammanee, K., 2009), we have

$$
\begin{aligned}
& \sum_{i, k} \sum_{\substack{l, m \\
(l, m) \neq(i, k)}} E Y\left(i, \rho_{1}(k), \ldots, \rho_{d-1}(k)\right) Y\left(l, \rho_{1}(m), \ldots, \rho_{d-1}(m)\right) \\
& =\sum_{i=1}^{n} \sum_{k=1}^{n} \sum_{\substack{l \\
l \neq i}} \sum_{m \neq k} E Y\left(i, \rho_{1}(k), \ldots, \rho_{d-1}(k)\right) Y\left(l, \rho_{1}(m), \ldots, \rho_{d-1}(m)\right) \\
& +\sum_{i=1}^{n} \sum_{k=1}^{n} \sum_{\substack{l \\
l \neq i}} E Y\left(i, \rho_{1}(k), \ldots, \rho_{d-1}(k)\right) Y\left(l, \rho_{1}(k), \ldots, \rho_{d-1}(k)\right)
\end{aligned}
$$

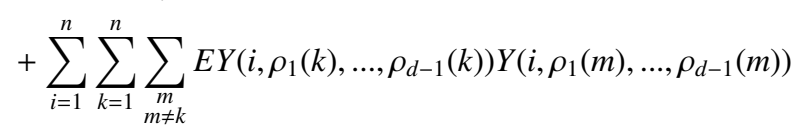

$$
\begin{aligned}
& =\frac{1}{(n(n-1))^{(d-2)}} \sum_{k_{1}=1}^{n} \ldots \sum_{k_{d}=1}^{n} E Y\left(k_{1}, \ldots, k_{d}\right) \sum_{\substack{m_{1} \\
m_{1} \neq k_{1}}} \ldots \sum_{\substack{m_{d} \\
m_{d} \neq k_{d}}} E Y\left(m_{1}, \ldots, m_{d}\right) \\
& +\frac{1}{n^{(d-2)}} \sum_{k_{1}=1}^{n} \ldots \sum_{k_{d}=1}^{n} E Y\left(k_{1}, \ldots, k_{d}\right) \sum_{\substack{l \\
l \neq k_{1}}} E Y\left(l, k_{2}, \ldots, k_{d}\right) \\
& +\frac{1}{(n(n-1))^{(d-2)}} \sum_{k_{1}=1}^{n} \ldots \sum_{k_{d}=1}^{n} E Y\left(k_{1}, \ldots, k_{d}\right) \sum_{\substack{m_{2} \\
m_{2} \neq k_{2}}} \ldots \sum_{\substack{m_{d} \\
m_{d} \neq k_{d}}} E Y\left(k_{1}, m_{2}, \ldots, m_{d}\right) \\
& \leq \frac{C}{n^{d-2}} \sum_{k_{1}=1}^{n} \ldots \sum_{k_{d}=1}^{n}\left[E Y\left(k_{1}, \ldots, k_{d}\right)\right]^{2} \\
& =C n \sqrt{n} \delta_{2} \text {. }
\end{aligned}
$$


Thus

$$
\begin{aligned}
E\left[\sum_{i=1}^{n} \sum_{k=1}^{n} Y\left(i, \rho_{1}(k), \ldots, \rho_{d-1}(k)\right)\right]^{2} & \leq \sum_{i=1}^{n} \sum_{k=1}^{n} E Y^{2}\left(i, \rho_{1}(k), \ldots, \rho_{d-1}(k)\right)+C n \sqrt{n} \delta_{2} \\
& =C n \sqrt{n} \delta_{2} .
\end{aligned}
$$

Using the fact that $2 a b \leq a^{2}+b^{2}$ and

$$
\begin{gathered}
E\left|Y\left(i_{1}, \ldots, i_{d}\right)\right|^{m}\left|Y_{z}\left(i_{1}, \ldots, i_{d}\right)\right|^{n} \\
\leq E\left|Y\left(i_{1}, \ldots, i_{d}\right)\right|^{m}\left|Y_{z}\left(i_{1}, \ldots, i_{d}\right)\right|^{n}\left(\frac{\left|Y_{z}\left(i_{1}, \ldots, i_{d}\right)\right|}{1+z}\right)^{t} \\
\leq \frac{E\left|Y\left(i_{1}, \ldots, i_{d}\right)\right|^{m+n+t}}{(1+z)^{t}}
\end{gathered}
$$

for any integers $m, n$ and $t$ which $m \geq 0, n, t>0$,

we have

$$
\begin{aligned}
& \sum_{i, k} \sum_{\substack{l, m \\
(l, m) \neq(i, k)}} E Y_{z}\left(i, \rho_{1}(k), \ldots, \rho_{d-1}(k)\right) Y_{z}\left(l, \rho_{1}(m), \ldots, \rho_{d-1}(m)\right) \\
& =\frac{1}{(n(n-1))^{(d-2)}} \sum_{k_{1}=1}^{n} \ldots \sum_{k_{d}=1}^{n} E Y_{z}\left(k_{1}, \ldots, k_{d}\right) \sum_{\substack{m_{1} \\
m_{1} \neq k_{1}}} \ldots \sum_{\substack{m_{d} \\
m_{d} \neq k_{d}}} E Y_{z}\left(m_{1}, \ldots, m_{d}\right) \\
& +\frac{1}{n^{(d-2)}} \sum_{k_{1}=1}^{n} \ldots \sum_{k_{d}=1}^{n} E Y_{z}\left(k_{1}, \ldots, k_{d}\right) \sum_{\substack{l \\
l \neq k_{1}}} E Y_{z}\left(l, k_{2}, \ldots, k_{d}\right) \\
& +\frac{1}{(n(n-1))^{(d-2)}} \sum_{k_{1}=1}^{n} \ldots \sum_{k_{d}=1}^{n} E Y_{z}\left(k_{1}, \ldots, k_{d}\right) \sum_{\substack{m_{2} \\
m_{2} \neq k_{2}}} \ldots \sum_{\substack{m_{d} \\
m_{d} \neq k_{d}}} E Y_{z}\left(k_{1}, m_{2}, \ldots, m_{d}\right) \\
& \leq \frac{C}{n^{2(d-2)}}\left[\sum_{k_{1}=1}^{n} \ldots \sum_{k_{d}=1}^{n} E\left|Y_{z}\left(k_{1}, \ldots, k_{d}\right)\right|\right]^{2}+\frac{C}{n^{d-3}} \sum_{k_{1}=1}^{n} \ldots \sum_{k_{d}=1}^{n} E Y_{z}^{2}\left(k_{1}, \ldots, k_{d}\right) \\
& \leq \frac{C}{n^{2(d-2)}}\left[\sum_{k_{1}=1}^{n} \ldots \sum_{k_{d}=1}^{n} \frac{E Y^{4}\left(k_{1}, \ldots, k_{d}\right)}{(1+z)^{3}}\right]^{2}+\frac{C}{n^{d-3}} \sum_{k_{1}=1}^{n} \ldots \sum_{k_{d}=1}^{n} \frac{E Y^{4}\left(k_{1}, \ldots, k_{d}\right)}{(1+z)^{2}} \\
& \leq C\left(n \delta_{4}^{2}+n \sqrt{n} \delta_{4}\right) .
\end{aligned}
$$

Thus

$$
\begin{aligned}
& E\left[\sum_{i=1}^{n} \sum_{k=1}^{n} Y_{z}\left(i, \rho_{1}(k), \ldots, \rho_{d-1}(k)\right)\right]^{2} \\
\leq & \sum_{i=1}^{n} \sum_{k=1}^{n} E Y_{z}^{2}\left(i, \rho_{1}(k), \ldots, \rho_{d-1}(k)\right)+C\left(n \delta_{4}^{2}+n \sqrt{n} \delta_{4}\right) \\
= & C\left(n \sqrt{n} \delta_{2}+n \delta_{4}^{2}+n \sqrt{n} \delta_{4}\right) .
\end{aligned}
$$

Now, we conclude from (2.2) and (2.4) that

$$
\begin{aligned}
& E\left[\sum_{i=1}^{n} \sum_{k=1}^{n} \widehat{Y}_{z}\left(i, \rho_{1}(k), \ldots, \rho_{d-1}(k)\right)\right]^{2} \\
& =E\left[\sum_{i=1}^{n} \sum_{k=1}^{n} Y\left(i, \rho_{1}(k), \ldots, \rho_{d-1}(k)\right)-\sum_{i=1}^{n} \sum_{k=1}^{n} Y_{z}\left(i, \rho_{1}(k), \ldots, \rho_{d-1}(k)\right)\right]^{2} \\
& \leq 2 E\left[\sum_{i=1}^{n} \sum_{k=1}^{n} Y\left(i, \rho_{1}(k), \ldots, \rho_{d-1}(k)\right)\right]^{2}+2 E\left[\sum_{i=1}^{n} \sum_{k=1}^{n} Y_{z}\left(i, \rho_{1}(k), \ldots, \rho_{d-1}(k)\right)\right]^{2} \\
& \leq C\left(n \sqrt{n} \delta_{2}+n \delta_{4}^{2}+n \sqrt{n} \delta_{4}\right) \\
& \leq C\left(n^{\frac{5}{4}} \delta_{4}^{\frac{1}{2}}+n \delta_{4}^{2}+n \sqrt{n} \delta_{4}\right) \\
& \leq C\left(n^{\frac{5}{4}} \delta_{4}^{\frac{1}{2}}+n^{2} \delta_{4}^{2}\right)
\end{aligned}
$$


where we have used the fact that

$$
\delta_{2}^{2}=\frac{1}{n^{2 d-1}}\left[\sum_{i_{1}=1}^{n} \ldots \sum_{i_{d}=1}^{n} E Y^{2}\left(i_{1}, \ldots, i_{d}\right)\right]^{2} \leq \frac{1}{n^{d-1}} \sum_{i_{1}=1}^{n} \ldots \sum_{i_{d}=1}^{n} E Y^{4}\left(i_{1}, \ldots, i_{d}\right)=\frac{\delta_{4}}{\sqrt{n}}
$$

in the third inequality and $n \sqrt{n} \delta_{4} \leq n^{\frac{5}{4}} \delta_{4}^{\frac{1}{2}}+n^{2} \delta_{4}^{2}$ in the last inequality.

Lemma 2.2 Suppose $E Y^{4}\left(i_{1}, \ldots, i_{d}\right)<\infty, 1 \leq i_{1}, \ldots, i_{d} \leq n$. Then

$$
\widehat{E Y}^{4}(\rho) \leq C\left(\sqrt{n} \delta_{4}+\delta_{4}^{2}\right)
$$

proof We observe that

$$
E\left(\sum_{i=1}^{n} Y_{z}\left(i, \rho_{1}(i), \ldots, \rho_{d-1}(i)\right)^{4}=M_{1}+M_{2}+M_{3}+M_{4}+M_{5}\right.
$$

where

$$
\begin{aligned}
M_{1}= & \sum_{i=1}^{n} E Y_{z}^{4}\left(i, \rho_{1}(i), \ldots, \rho_{d-1}(i)\right), \\
M_{2}= & \sum_{i=1}^{n} \sum_{\substack{j \\
j \neq i}} E Y_{z}\left(i, \rho_{1}(i), \ldots, \rho_{d-1}(i)\right) Y_{z}^{3}\left(j, \rho_{1}(j), \ldots, \rho_{d-1}(j)\right), \\
M_{3}= & \sum_{i=1}^{n} \sum_{\substack{j \\
j \neq i}} E Y_{z}^{2}\left(i, \rho_{1}(i), \ldots, \rho_{d-1}(i)\right) Y_{z}^{2}\left(j, \rho_{1}(j), \ldots, \rho_{d-1}(j)\right), \\
M_{4}= & \sum_{i=1}^{n} \sum_{\substack{j \\
j \neq i}} \sum_{\substack{k \\
k \neq i, j}} E Y_{z}^{2}\left(i, \rho_{1}(i), \ldots, \rho_{d-1}(i)\right) Y_{z}\left(j, \rho_{1}(j), \ldots, \rho_{d-1}(j)\right) \\
& Y_{z}\left(k, \rho_{1}(k), \ldots, \rho_{d-1}(k)\right) \text { and } \\
M_{5}= & \sum_{i=1}^{n} \sum_{\substack{j \\
j \neq i}} \sum_{\substack{k \\
k \neq i, j}} \sum_{l \neq i, j, k} E Y_{z}\left(i, \rho_{1}(i), \ldots, \rho_{d-1}(i)\right) Y_{z}\left(j, \rho_{1}(j), \ldots, \rho_{d-1}(j)\right) \\
& Y_{z}\left(k, \rho_{1}(k), \ldots, \rho_{d-1}(k)\right) Y_{z}\left(l, \rho_{1}(l), \ldots, \rho_{d-1}(l)\right) .
\end{aligned}
$$

We note that

$$
\left|M_{1}\right|=\frac{1}{n^{d-1}} \sum_{i_{1}=1}^{n} \ldots \sum_{i_{d}=1}^{n} E Y_{z}^{4}\left(i_{1}, \ldots, i_{d}\right)=\frac{\delta_{4}}{\sqrt{n}}
$$

It follows from (2.3) and (2.5)that

$$
\begin{aligned}
\left|M_{2}\right| & \leq \frac{C}{n^{2(d-1)}} \sum_{i_{1}=1}^{n} \ldots \sum_{i_{d}=1}^{n} E\left|Y_{z}\left(i_{1}, \ldots, i_{d}\right)\right| \sum_{j_{1}=1}^{n} \ldots \sum_{j_{d}=1}^{n} E\left|Y_{z}\left(j_{1}, \ldots, j_{d}\right)\right|^{3} \\
& \leq \frac{C}{n^{2(d-1)}}\left[\sum_{i_{1}=1}^{n} \ldots \sum_{i_{d}=1}^{n} E Y^{4}\left(i_{1}, \ldots, i_{d}\right)\right]^{2} \\
& =\frac{C \delta_{4}^{2}}{n}
\end{aligned}
$$




$$
\begin{aligned}
\left|M_{3}\right| & \leq \frac{C}{n^{2(d-1)}} \sum_{i_{1}=1}^{n} \ldots \sum_{i_{d}=1}^{n} \sum_{j_{1}=1}^{n} \ldots \sum_{j_{d}=1}^{n} E Y_{z}^{2}\left(i_{1}, \ldots, i_{d}\right) E Y_{z}^{2}\left(j_{1}, \ldots, j_{d}\right) \\
& \leq \frac{C}{n^{2(d-1)}}\left[\sum_{i_{1}=1}^{n} \ldots \sum_{i_{d}=1}^{n} E Y^{4}\left(i_{1}, \ldots, i_{d}\right)\right]^{2} \\
& =\frac{C \delta_{4}^{2}}{n}, \\
\left|M_{4}\right| & \leq \frac{C}{n^{3(d-1)}} \sum_{i_{1}=1}^{n} \ldots \sum_{i_{d}=1}^{n} E Y_{z}^{2}\left(i_{1}, \ldots, i_{d}\right)\left[\sum_{j_{1}=1}^{n} \ldots \sum_{j_{d}=1}^{n} E\left|Y_{z}\left(j_{1}, \ldots, j_{d}\right)\right|\right]^{2} \\
& =\frac{C}{n^{3(d-1)}} \sum_{i_{1}=1}^{n} \ldots \sum_{i_{d}=1}^{n} E Y^{4}\left(i_{1}, \ldots, i_{d}\right)\left[\sum_{j_{1}=1}^{n} \ldots \sum_{j_{d}=1}^{n} E\left|Y\left(j_{1}, \ldots, j_{d}\right)\right|^{2}\right]^{2} \\
& =C \sqrt{n} \delta_{4} \delta_{2}^{2} \\
& \leq C \delta_{4}^{2} \text { and } \\
\left|M_{5}\right| & \leq \frac{C}{n^{4(d-1)}}\left[\sum_{i_{1}=1}^{n} \ldots \sum_{i_{d}=1}^{n} E\left|Y_{z}\left(i_{1}, \ldots, i_{d}\right)\right|\right]^{4} \\
& \leq \frac{C}{n^{4(d-1)}}\left[\sum_{i_{1}=1}^{n} \ldots \sum_{i_{d}=1}^{n} E Y^{2}\left(i_{1}, \ldots, i_{d}\right)\right]^{4} \\
& =C \delta_{2}^{4} \\
& \leq \frac{C \delta_{4}^{2}}{n} .
\end{aligned}
$$

Thus

$$
E\left(\sum_{i=1}^{n} Y_{z}\left(i, \rho_{1}(i), \ldots, \rho_{d-1}(i)\right)\right)^{4} \leq C\left(\frac{\delta_{4}}{\sqrt{n}}+\delta_{4}^{2}\right)
$$

By this fact and $E W^{4} \leq C \sqrt{n} \delta_{4}$ (Neammanee, K., 2008),

$$
\begin{aligned}
E \widehat{Y}^{4}(\rho) & =E\left(\sum_{i=1}^{n} Y\left(i, \rho_{1}(i), \ldots, \rho_{d-1}(i)\right)-\sum_{i=1}^{n} Y_{z}\left(i, \rho_{1}(i), \ldots, \rho_{d-1}(i)\right)\right)^{4} \\
& \leq E W^{4}+E\left(\sum_{i=1}^{n} Y_{z}\left(i, \rho_{1}(i), \ldots, \rho_{d-1}(i)\right)\right)^{4} \\
& \leq C\left(\sqrt{n} \delta_{4}+\delta_{4}^{2}\right) .
\end{aligned}
$$

\section{Lemma 2.3}

$$
E(\widetilde{Y}(\rho)-\widehat{Y}(\rho))^{2}=\frac{4}{n}+\Delta
$$

where $|\Delta| \leq C\left(\frac{\delta_{4}^{\frac{1}{2}}}{n^{\frac{5}{4}}}+\frac{\delta_{4}}{n}\right)$.

proof Let

$$
\begin{array}{ll}
S_{1}=Y\left(I, \rho_{1}(I), \ldots, \rho_{d-1}(I)\right), & S_{1, z}=Y_{z}\left(I, \rho_{1}(I), \ldots, \rho_{d-1}(I)\right), \\
S_{2}=Y\left(K, \rho_{1}(K), \ldots, \rho_{d-1}(K)\right), & S_{2, z}=Y_{z}\left(K, \rho_{1}(K), \ldots, \rho_{d-1}(K)\right) \\
S_{3}=Y\left(I, \rho_{1}(K), \ldots, \rho_{d-1}(K)\right), & S_{3, z}=Y_{z}\left(I, \rho_{1}(K), \ldots, \rho_{d-1}(K)\right), \text { and } \\
S_{4}=Y\left(K, \rho_{1}(I), \ldots, \rho_{d-1}(I)\right), & S_{4, z}=Y_{z}\left(K, \rho_{1}(I), \ldots, \rho_{d-1}(I)\right) .
\end{array}
$$

We observe that

$$
E(\widetilde{Y}(\rho)-\widehat{Y}(\rho))^{2}=\sum_{k=1}^{4} E S_{k}^{2}+\Delta_{1}
$$


where

$$
\left|\Delta_{1}\right| \leq \sum_{1 \leq i<j \leq 4}\left|E S_{i} S_{j}\right|+4 \sum_{i=1}^{4} E S_{i, z}^{2}+\sum_{i=1}^{n} \sum_{j=1}^{n} E\left|S_{i} S_{j, z}\right|
$$

and, by (2.1),

$$
\begin{aligned}
E S_{1}^{2} & =\frac{1}{n} \sum_{i=1}^{n} E Y^{2}\left(i, \rho_{1}(i), \ldots, \rho_{d-1}(i)\right) \\
& =\frac{1}{n}\left[E W^{2}-\sum_{i=1}^{n} \sum_{\substack{j \\
j \neq i}} E Y\left(i, \rho_{1}(i), \ldots, \rho_{d-1}(i)\right) Y\left(j, \rho_{1}(j), \ldots, \rho_{d-1}(j)\right)\right] \\
& =\frac{1}{n}-\frac{1}{n^{d}(n-1)^{d-1}} \sum_{i_{1}=1}^{n} \ldots \sum_{i_{d}=1}^{n} \sum_{\substack{j_{1} \\
j_{1} \neq i_{1}}} \ldots \sum_{\substack{j_{d} \\
j_{d} \neq i_{d}}} E Y\left(i_{1}, \ldots, i_{d}\right) E Y\left(j_{1}, \ldots, j_{d}\right) \\
& =\frac{1}{n}-\frac{(-1)^{d}}{n^{d}(n-1)^{d-1}} \sum_{i_{1}=1}^{n} \ldots \sum_{i_{d}=1}^{n}\left[E Y\left(i_{1}, \ldots, i_{d}\right)\right]^{2} .
\end{aligned}
$$

Thus, from (2.6), (2.7) and the fact that $S_{1}, S_{2}, S_{3}, S_{4}$ have the same distribution, we have

$$
E(\widetilde{Y}(\rho)-\widehat{Y}(\rho))^{2}=\frac{4}{n}+\Delta_{2}
$$

where

$$
\left|\Delta_{2}\right| \leq \frac{C \delta_{2}}{n \sqrt{n}}+\sum_{1 \leq i<j \leq 4}\left|E S_{i} S_{j}\right|+4 \sum_{i=1}^{4} E S_{i, z}^{2}+\sum_{i=1}^{n} \sum_{j=1}^{n} E\left|S_{i} S_{j, z}\right| .
$$

To prove the lemma, it suffices to find appropriate bounds the right-hand side of (2.8). We note from (2.3) that

$$
\begin{aligned}
E S_{1, z}^{2} & =\frac{1}{n} \sum_{i=1}^{n} E Y_{z}^{2}\left(i, \rho_{1}(i), \ldots, \rho_{d-1}(i)\right) \\
& =\frac{1}{n^{d}} \sum_{i_{1}=1}^{n} \ldots \sum_{i_{d}=1}^{n} E Y_{z}^{2}\left(i_{1}, \ldots, i_{d}\right) \\
& \leq \frac{C}{n^{d}} \sum_{i_{1}=1}^{n} \ldots \sum_{i_{d}=1}^{n} E Y^{4}\left(i_{1}, \ldots, i_{d}\right) \\
& =\frac{C \delta_{4}}{n \sqrt{n}} .
\end{aligned}
$$

From this fact, (2.5) and the fact that $E\left|S_{1}\right|^{k} \leq \frac{\delta_{k}}{n^{\frac{k-1}{2}}}$ for $k \in \mathbb{N}$, we have

$$
E\left|S_{i} S_{j, z}\right| \leq\left\{E S_{i}^{2}\right\}^{\frac{1}{2}}\left\{E S_{j, z}^{2}\right\}^{\frac{1}{2}}=\left\{E S_{1}^{2}\right\}^{\frac{1}{2}}\left\{E S_{1, z}^{2}\right\}^{\frac{1}{2}} \leq \frac{C \delta_{2}^{\frac{1}{2}} \delta_{4}^{\frac{1}{2}}}{n} \leq \frac{C \delta_{4}^{\frac{3}{4}}}{n^{\frac{9}{8}}}
$$

for $i, j=1,2,3,4$. Note from $(2.1)$ that

$$
\begin{aligned}
\left|E S_{1} S_{2}\right| & =\left|E Y\left(I, \rho_{1}(I), \ldots, \rho_{d-1}(I)\right) Y\left(K, \rho_{1}(K), \ldots, \rho_{d-1}(K)\right)\right| \\
& =\left|\frac{1}{n(n-1)} \sum_{i=1}^{n} \sum_{\substack{k \\
k \neq i}} E Y\left(i, \rho_{1}(i), \ldots, \rho_{d-1}(i)\right) Y\left(k, \rho_{1}(k), \ldots, \rho_{d-1}(k)\right)\right| \\
& =\left|\frac{1}{(n(n-1))^{d}} \sum_{i_{1}=1}^{n} \ldots \sum_{i_{d}=1}^{n} E Y\left(i_{1}, \ldots, i_{d}\right) \sum_{\substack{k_{1} \\
k_{1} \neq i_{1}}} \ldots \sum_{\substack{k_{d} \\
k_{d} \neq i_{d}}} E Y\left(k_{1}, \ldots, k_{d}\right)\right| \\
& =\left|\frac{1}{(n(n-1))^{d}} \sum_{i_{1}=1}^{n} \ldots \sum_{i_{d}=1}^{n}\left(E Y\left(i_{1}, \ldots, i_{d}\right)\right)^{2}\right| \\
& =\frac{C \delta_{2}}{n \sqrt{n}} .
\end{aligned}
$$


By the same argument, we can show that

$$
\left|E S_{i} S_{j}\right| \leq \frac{C \delta_{2}}{n \sqrt{n}}
$$

for $1 \leq i<j \leq 4$. Now we conclude from (2.8) - (2.11) that

$$
|\Delta| \leq C\left(\frac{\delta_{2}}{n \sqrt{n}}+\frac{\delta_{4}^{\frac{3}{4}}}{n^{\frac{9}{8}}}+\frac{\delta_{4}}{n \sqrt{n}}\right) \leq C\left(\frac{\delta_{4}^{\frac{1}{2}}}{n^{\frac{7}{4}}}+\frac{\delta_{4}^{\frac{3}{4}}}{n^{\frac{9}{8}}}+\frac{\delta_{4}}{n \sqrt{n}}\right) \leq C\left(\frac{\delta_{4}^{\frac{1}{2}}}{n^{\frac{5}{4}}}+\frac{\delta_{4}}{n}\right) .
$$

\section{Proof of Theorem 1.2}

To bound $|P(W \leq z)-\Phi(z)|$, it suffices to consider $z \geq 0$ as we have used the fact that $\Phi(z)=1-\Phi(-z)$ and apply the result to $-W$ when $z<0$. So, from now on, we assume $z \geq 0$.

Note that

$$
|P(W \leq z)-\Phi(z)| \leq P(W \neq \widehat{Y}(\rho))+|P(\widehat{Y}(\rho) \leq z)-\Phi(z)|
$$

and

$$
\begin{aligned}
P(W \neq \widehat{Y}(\rho)) & =P\left(\sum_{i=1}^{n} \mathbb{I}\left(\left|Y\left(i, \rho_{1}(i), \ldots, \rho_{d-1}(i)\right)\right|>1+z\right) \geq 1\right) \\
& \leq \sum_{i=1}^{n} E \mathbb{I}\left(\left|Y\left(i, \rho_{1}(i), \ldots, \rho_{d-1}(i)\right)\right|>1+z\right) \\
& =\frac{1}{n^{d-1}} \sum_{i_{1}=1}^{n} \ldots \sum_{i_{d}=1}^{n} E \mathbb{I}\left(\left|Y\left(i_{1}, \ldots, i_{d}\right)\right|>1+z\right) \\
& =\frac{1}{n^{d-1}} \sum_{i_{1}=1}^{n} \ldots \sum_{i_{d}=1}^{n} P\left(\left|Y\left(i_{1}, \ldots, i_{d}\right)\right|>1+z\right) \\
& \leq \frac{1}{n^{d-1}} \sum_{i_{1}=1}^{n} \ldots \sum_{i_{d}=1}^{n} \frac{E\left|Y\left(i_{1}, \ldots, i_{d}\right)\right|^{3}}{(1+z)^{3}} \\
& =\frac{\delta_{3}}{(1+z)^{3}} .
\end{aligned}
$$

Hence, it suffices to bound $|P(\widehat{Y}(\rho) \leq z)-\Phi(z)|$. To do this we will apply Stein's method and the idea from Neammanee and Rattanawong(Neammanee, K., 2008).

In 1972, Stein(Stein, C.M., 1972) introduced a powerful and general method for obtaining explicit bound for the error in the normal approximation to the distribution of a sum of dependent random variables. His technique was relied instead on the elementary differantial equation. The Stein's equation for normal distribution function is

$$
g^{\prime}(w)-w g(w)=\mathbb{I}(w \leq z)-\Phi(z) \text { for } w \in \mathbb{R} .
$$

It is well-known that the solution $g_{z}$ of (3.3) is of the form

$$
\begin{aligned}
& \qquad g_{z}(w)=\left\{\begin{array}{l}
\sqrt{2 \pi} e^{\frac{1}{2} w^{2}} \Phi(w)[1-\Phi(z)] \quad \text { if } \quad w \leq z, \\
\sqrt{2 \pi} e^{\frac{1}{2} w^{2}} \Phi(z)[1-\Phi(w)] \quad \text { if } w>z,
\end{array}\right. \\
& \text { with } \quad 0 \leq g_{z}(w) \leq 1 \text { for all } w \in \mathbb{R}, \\
& \qquad<g_{z}(w) \leq \min \left(\frac{\sqrt{2 \pi}}{4}, \frac{1}{|z|}\right) \text { for all } w \in \mathbb{R} \text { and } z \neq 0, \\
& \quad\left|g_{z}^{\prime}(w)-g_{z}^{\prime}(v)\right| \leq 1, \text { for all real } w, v, \\
& \text { and } \quad\left|g_{z}^{\prime}(w)\right| \leq 1 \text { for all } w \in \mathbb{R}
\end{aligned}
$$

(Stein(1972, pp.22-23)).

From (3.3),

$$
|P(\widehat{Y}(\rho) \leq z)-\Phi(z)|=\left|E g_{z}^{\prime}(\widehat{Y}(\rho))-E \widehat{Y}(\rho) g_{z}(\widehat{Y}(\rho))\right| .
$$

To bound the right handside of (3.7), Neammanee and Rattanawong(2008) construct the random permutation $\tau_{1}, \tau_{2}, \ldots, \tau_{d-1}$ in the followings. 
Let $I, K, L_{1}, \ldots, L_{d-1}, M_{1}, \ldots, M_{d-1}$ be random variables with uniformly distribution on $\{1,2, \ldots, n\}$ and $\rho_{1}, \ldots, \rho_{d-1}, \tau_{1}, \ldots, \tau_{d-1}$ are random permutations of $\{1,2, \ldots, n\}$. Assume that

$\left\{I, K, L_{1}, \ldots, L_{d-1}, M_{1}, \ldots, M_{d-1}, \rho_{1}, \ldots, \rho_{d-1}, \tau_{1}, \ldots, \tau_{d-1}\right\}$ is independent of $Y\left(i_{1}, \ldots, i_{d}\right)^{\prime} \mathrm{s}$,

$(I, K),\left(L_{1}, M_{1}\right), \ldots,\left(L_{d-1}, M_{d-1}\right)$ are uniformly distributed on

$\{(i, k) \mid i, k=1,2, \ldots, n$ and $i \neq k$,

$(I, K),\left(L_{1}, M_{1}\right), \ldots,\left(L_{d-1}, M_{d-1}\right)$ and $\tau_{1}, \ldots, \tau_{d-1}$ are mutually independent,

$(I, K)$ and $\rho_{1}, \ldots, \rho_{d-1}$ are mutually independent, and

$$
\rho_{i}(\alpha)= \begin{cases}\tau_{i}(\alpha) & \text { if } \quad \alpha \neq I, K, \tau_{i}^{-1}\left(L_{i}\right), \tau_{i}^{-1}\left(M_{i}\right), \\ L_{i} & \text { if } \quad \alpha=I, \\ M_{i} & \text { if } \quad \alpha=K, \\ \tau_{i}(I) & \text { if } \quad \alpha=\tau_{i}^{-1}\left(L_{i}\right), \\ \tau_{i}(K) & \text { if } \quad \alpha=\tau_{i}^{-1}\left(M_{i}\right),\end{cases}
$$

where $\rho_{i}\left(\rho_{i}^{-1}(\alpha)\right)=\rho_{i}^{-1}\left(\rho_{i}(\alpha)\right)=\alpha$, for $i=1, \ldots, d-1$.

Then, they showed that

$$
\begin{aligned}
\left.\mid g_{z}^{\prime} \widehat{Y}(\rho)\right)-\widehat{Y}(\rho) g_{z}(\widehat{Y}(\rho)) \mid \leq & \left|E g_{z}^{\prime}(\widehat{Y}(\tau)) \int_{-\infty}^{\infty} K(t) d t-E \int_{-\infty}^{\infty} g_{z}^{\prime}(\widehat{Y}(\rho)+t) K(t) d t\right| \\
& +\left|E g_{z}^{\prime}(\widehat{Y}(\tau)) E \int_{-\infty}^{\infty} K(t) d t-E g_{z}^{\prime}(\widehat{Y}(\tau)) \int_{-\infty}^{\infty} K(t) d t\right| \\
& +\left|E g_{z}^{\prime}(\widehat{Y}(\tau))-E g_{z}^{\prime}(\widehat{Y}(\tau)) E \int_{-\infty}^{\infty} K(t) d t\right| \\
& +\left|\Delta g_{z}(\widehat{Y}(\rho))\right| \\
: & =\left|T_{1}\right|+\left|T_{2}\right|+\left|T_{3}\right|+\left|T_{4}\right| .
\end{aligned}
$$

We observe from Lemma 2.1 and (3.4) that

$$
\left|T_{4}\right| \leq C\left\{\frac{\delta_{4}^{\frac{1}{4}}}{n^{\frac{3}{8}}}+\delta_{4}\right\}\left\{E g_{z}^{2}(\widehat{Y}(\rho))\right\}^{\frac{1}{2}} \leq \frac{C}{1+z}\left\{\frac{\delta_{4}^{\frac{1}{4}}}{n^{\frac{3}{8}}}+\delta_{4}\right\} .
$$

By the fact that

$$
\left|g_{z}^{\prime}(w+s)-g_{z}^{\prime}(w+t)-\int_{t}^{s} h(w+u) d u\right| \leq \mathbb{I}(z-\max (s, t)<w<z-\min (s, t))
$$

(Chen, L.H.Y., 2001), we have

$$
\begin{aligned}
\left|T_{1}\right| & \leq E \int_{-\infty}^{\infty}\left|g_{z}^{\prime}(\widehat{Y}(\tau))-g_{z}^{\prime}(\widehat{Y}(\rho)+t)\right| K(t) d t \\
& =E \int_{-\infty}^{\infty}\left|g_{z}^{\prime}(\widehat{Y}(\rho)+\Delta \widehat{Y})-g_{z}^{\prime}(\widehat{Y}(\rho)+t)\right| K(t) d t \\
& \leq T_{11}+T_{12}+T_{13}
\end{aligned}
$$

where

$$
\begin{aligned}
\Delta \widehat{Y} & =\widehat{Y}(\tau)-\widehat{Y}(\rho) \\
T_{11} & =E \mathbb{I}\left(\delta<\frac{z}{4}\right) \int_{-\infty}^{\infty} \mathbb{I}(z-\max (\Delta \widehat{Y}, t)<\widehat{Y}(\rho)<z-\min (\Delta \widehat{Y}, t)) K(t) d t \\
T_{12} & =E \mathbb{I}\left(\delta<\frac{z}{4}\right) \int_{-\infty}^{\infty} \int_{t}^{\Delta \widehat{Y}} h(\widehat{Y}(\rho)+u) K(t) d u d t \\
T_{13} & =E \mathbb{I}\left(\delta \geq \frac{z}{4}\right) \int_{-\infty}^{\infty}\left|g_{z}^{\prime}(\widehat{Y}(\rho)+\Delta \widehat{Y})-g_{z}^{\prime}(\widehat{Y}(\rho)+t)\right| K(t) d t \\
\delta & =|\Delta \widehat{Y}|+|\widetilde{Y}(\rho)-\widehat{Y}(\rho)| \text { and } \\
h(w) & =\left(w g_{z}(w)\right)^{\prime} .
\end{aligned}
$$

Hence, by (3.1), (3.2), (3.7)-(3.10),

$$
|P(W \leq z)-\Phi(z)| \leq T_{11}+T_{12}+T_{13}+\left|T_{2}\right|+\left|T_{3}\right|+\frac{C}{1+z}\left(\frac{\delta_{4}^{\frac{1}{4}}}{n^{\frac{3}{8}}}+\delta_{3}+\delta_{4}\right) .
$$


Step 1. $T_{11} \leq \frac{C}{1+z}\left(\frac{\delta_{4}^{\frac{1}{4}}}{n^{\frac{3}{8}}}+n \delta_{4}^{\frac{7}{2}}\right)$

We used the idea from Chen and Shao(Chen, L.H.Y., 2001) to define $f_{\delta}: \mathbb{R} \rightarrow \mathbb{R}$ by

$$
f_{\delta}(t)= \begin{cases}0 & \text { if } t<z-2 \delta \\ (1+t+\delta)(t-z+2 \delta) & \text { if } z-2 \delta \leq t \leq z+2 \delta \\ 4 \delta(1+t+\delta) & \text { if } t>z+2 \delta\end{cases}
$$

Note that

$$
\left|f_{\delta}(t)\right| \leq 4 \delta(1+|t|+\delta) \quad \text { for all } t
$$

and

$$
f_{\delta}^{\prime}(t) \geq \begin{cases}1+z-\delta & \text { if } z-2 \delta \leq t \leq z+2 \delta \\ 0 & \text { otherwise. }\end{cases}
$$

By the same argument as in Lemma 2.1, we have

$$
E \int_{-\infty}^{\infty} f_{\delta}^{\prime}(\widehat{Y}(\rho)+t) K(t) d t \leq E \widehat{Y}(\rho) f_{\delta}(\widehat{Y}(\rho))+C\left(\frac{\delta_{4}^{\frac{1}{4}}}{n^{\frac{3}{8}}}+\delta_{4}\right)\left(E f_{\delta}^{2}(\widehat{Y}(\rho))\right)^{\frac{1}{2}}
$$

Thus, by (3.13) and (3.14),

$$
\begin{aligned}
& T_{11} \leq E \int_{|t| \leq|\widetilde{Y}(\rho)-\widehat{Y}(\rho)|} \mathbb{I}\left(\delta<\frac{z}{4}\right) \mathbb{I}(z-(|\Delta \widehat{Y}|+|t|)<\widehat{Y}(\rho)<z+(|\Delta \widehat{Y}|+|t|)) K(t) d t \\
& \leq E \int_{|t| \leq|\widetilde{Y}(\rho)-\widehat{Y}(\rho)|} \mathbb{I}\left(\delta<\frac{z}{4}\right) \mathbb{I}(z-\delta<\widehat{Y}(\rho)<z+\delta) K(t) d t \\
& \leq \frac{C}{1+z} E \int_{|t| \leq|\widetilde{Y}(\rho)-\widehat{Y}(\rho)|} \mathbb{I}\left(\delta<\frac{z}{4}\right)(1+z-\delta) \mathbb{I}(z-\delta<\widehat{Y}(\rho)<z+\delta) K(t) d t \\
& \leq \frac{C}{1+z} E \int_{|t| \leq|\widetilde{Y}(\rho)-\widehat{Y}(\rho)|} f_{\delta}^{\prime}(\widehat{Y}(\rho)+t) K(t) d t \\
& \leq \frac{C}{1+z}\left[E \widehat{Y}(\rho) f_{\delta}(\widehat{Y}(\rho))+\left(\frac{\delta_{4}^{\frac{1}{4}}}{n^{\frac{3}{8}}}+\delta_{4}\right)\left(E f_{\delta}^{2}(\widehat{Y}(\rho))\right)^{\frac{1}{2}}\right] .
\end{aligned}
$$

To bound $E\left|\widehat{Y}(\rho) f_{\delta}(\widehat{Y}(\rho))\right|$ and $E f_{\delta}^{2}(\widehat{Y}(\rho))$, we note that

$$
\begin{aligned}
E \widehat{Y}^{2}(\rho) & =E\left[\sum_{i=1}^{n} \widehat{Y}_{z}\left(i, \rho_{1}(i), \ldots, \rho_{d-1}(i)\right)\right]^{2} \\
& =E\left[\sum_{i=1}^{n} Y\left(i, \rho_{1}(i), \ldots, \rho_{d-1}(i)\right)-\sum_{i=1}^{n} Y_{z}\left(i, \rho_{1}(i), \ldots, \rho_{d-1}(i)\right)\right]^{2} \\
& \leq E\left[\sum_{i=1}^{n} Y\left(i, \rho_{1}(i), \ldots, \rho_{d-1}(i)\right)\right]^{2}+E\left[\sum_{i=1}^{n} Y_{z}\left(i, \rho_{1}(i), \ldots, \rho_{d-1}(i)\right)\right]^{2} \\
& \leq E W^{2}+n \sum_{i=1}^{n} E Y_{z}^{2}\left(i, \rho_{1}(i), \ldots, \rho_{d-1}(i)\right) \\
& =1+\frac{1}{n^{(d-2)}} \sum_{i_{1}=1}^{n} \ldots \sum_{i_{d-1}=1}^{n} E Y_{z}^{2}\left(i_{1}, \ldots, i_{d}\right) \\
& \leq 1+\frac{1}{n^{(d-2)}} \sum_{i_{1}=1}^{n} \ldots \sum_{i_{d-1}=1}^{n} \frac{E Y^{4}\left(i_{1}, \ldots, i_{d}\right)}{(1+z)^{2}} \\
& =1+C \sqrt{n} \delta_{4}, \\
& E|\widetilde{Y}(\rho)-\widehat{Y}(\rho)|^{k} \leq C \sum_{i=1}^{4} E\left|\widehat{S}_{i, z}\right|^{k}=C E\left|\widehat{S}_{1, z}\right|^{k} \leq \frac{C \delta_{k}}{n^{\frac{k-1}{2}}}, \\
& E|\Delta \widehat{Y}|^{k} \leq \frac{C \delta_{k}}{n^{\frac{k-1}{2}}}
\end{aligned}
$$


and

$$
E \delta^{k} \leq \frac{C \delta_{k}}{n^{\frac{k-1}{2}}} \text { for all } k \in \mathbb{N} .
$$

We conclude from Lemma 2.2, (2.5), (3.12), (3.16) - (3.19) and Cauchy - Schwarz inequality,

$$
\begin{aligned}
E\left|\widehat{Y}(\rho) f_{\delta}(\widehat{Y}(\rho))\right| & \leq C\left(E|\widehat{Y}(\rho)| \delta+E \widehat{Y}^{2}(\rho) \delta+E|\widehat{Y}(\rho)| \delta^{2}\right) \\
& \leq C\left(\frac{\delta_{4}^{\frac{1}{4}}}{n^{\frac{3}{8}}}+\frac{\delta_{4}^{\frac{3}{4}}}{n^{\frac{1}{8}}}+\frac{\delta_{4}^{\frac{5}{4}}}{n^{\frac{3}{8}}}+\frac{\delta_{4}^{\frac{1}{2}}}{n^{\frac{3}{4}}}+\frac{\delta_{4}}{n^{\frac{1}{2}}}\right) \\
& \leq C\left(\frac{\delta_{4}^{\frac{1}{4}}}{n^{\frac{3}{8}}}+n^{\frac{1}{8}} \delta_{4}^{\frac{5}{4}}\right)
\end{aligned}
$$

and

$$
E f_{\delta}^{2}(\widehat{Y}(\rho)) \leq C E\left(\delta^{2}+\delta^{2} \widehat{Y}^{2}(\rho)+\delta^{4}\right) \leq \frac{\delta_{4}^{\frac{1}{2}}}{n^{\frac{3}{4}}}+\frac{\delta_{4}^{\frac{3}{2}}}{n^{\frac{1}{4}}} .
$$

From this fact and (3.15), $T_{11} \leq \frac{C}{1+z}\left(\frac{\delta_{4}^{\frac{1}{4}}}{n^{\frac{3}{8}}}+n \delta_{4}^{\frac{7}{2}}\right)$.

Step 2. $T_{12}+T_{13} \leq \frac{C}{1+z}\left(\delta_{3}+\delta_{4}^{\frac{1}{2}} \delta_{6}^{\frac{1}{2}}+\delta_{4} \delta_{6}^{\frac{1}{2}}\right)$.

Since

$$
\begin{aligned}
E \int_{-\infty}^{\infty} \int_{t}^{\Delta \widehat{Y}} K(t) d u d t & \leq E \int_{-\infty}^{\infty}(|\Delta \widehat{Y}|+|t|) K(t) d t \\
& \leq C n\left(E|\Delta \widehat{Y}|(\widetilde{Y}(\rho)-\widehat{Y}(\rho))^{2}+E|\widetilde{Y}(\rho)-\widehat{Y}(\rho)|^{3}\right) \\
& \leq C \delta_{3},
\end{aligned}
$$

and

$$
h(w) \leq \begin{cases}C(1+z) & \text { if } \frac{z}{2}<w \leq z \\ \frac{C}{(1+z)^{2}} & \text { if } w \leq \frac{z}{2} \text { or } w>z\end{cases}
$$

(Laipaporn, K., 2007),

$$
\begin{aligned}
& T_{12} \\
& \leq E \int_{-\infty}^{\infty} \int_{t}^{\Delta \widehat{Y}} h(\widehat{Y}(\rho)+u) K(t) \mathbb{I}\left(\widehat{Y}(\rho)+u \leq \frac{z}{2} \text { or } \widehat{Y}(\rho)+u>z\right) d u d t \\
& \quad+E \mathbb{I}\left(\delta<\frac{z}{4}\right) \int_{-\infty}^{\infty} \int_{t}^{\Delta \widehat{Y}} h(\widehat{Y}(\rho)+u) K(t) \mathbb{I}\left(\frac{z}{2}<\widehat{Y}(\rho)+u \leq z\right) d u d t \\
& \leq \frac{C \delta_{3}}{(1+z)^{2}}+C(1+z) E \mathbb{I}\left(\delta<\frac{z}{4}\right) \int_{-\infty}^{\infty} \int_{t}^{\Delta \widehat{Y}} K(t) \mathbb{I}\left(\widehat{Y}(\rho)+u>\frac{z}{2}\right) d u d t .
\end{aligned}
$$

It remains to bound the second term on the right hand side of (3.20). By the fact that $K(t)=0$ for $|t|>|\widehat{Y}(\rho)-\widetilde{Y}(\rho)|$, Lemma 2.2, (3.17) and (3.18),

$$
\begin{aligned}
& E \mathbb{I}\left(\delta<\frac{z}{4}\right) \int_{-\infty}^{\infty} \int_{t}^{\Delta \widehat{Y}} K(t) \mathbb{I}\left(\widehat{Y}(\rho)+u>\frac{z}{2}\right) d u d t \\
& \leq E \mathbb{I}\left(\delta<\frac{z}{4}\right) \int_{-\infty}^{\infty} \int_{t}^{\Delta \widehat{Y}} K(t) \mathbb{I}\left(\widehat{Y}(\rho)+\delta>\frac{z}{2}\right) d u d t \\
& =E \int_{-\infty}^{\infty} \int_{t}^{\Delta \widehat{Y}} K(t) \mathbb{I}\left(\widehat{Y}(\rho)>\frac{z}{4}\right) d u d t \\
& \leq E \mathbb{I}\left(\widehat{Y}(\rho)>\frac{z}{4}\right) \int_{-\infty}^{\infty}(|\Delta \widehat{Y}|+|t|) K(t) d t \\
& \leq \frac{n}{4}\left\{P\left(\widehat{Y}(\rho)>\frac{z}{4}\right)\right\}^{\frac{1}{2}}\left\{\left(E(\Delta \widehat{Y})^{2}(\widetilde{Y}(\rho)-\widehat{Y}(\rho))^{4}\right)^{\frac{1}{2}}+\left(E(\widetilde{Y}(\rho)-\widehat{Y}(\rho))^{6}\right)^{\frac{1}{2}}\right\}
\end{aligned}
$$




$$
\begin{aligned}
& \leq \frac{C}{z^{2}}\left(E \widehat{Y}^{4}(\rho)\right)^{\frac{1}{2}} \frac{\delta_{6}^{\frac{1}{2}}}{n^{\frac{1}{4}}} \\
& \leq \frac{C}{(1+z)^{2}}\left(\delta_{4}^{\frac{1}{2}} \delta_{6}^{\frac{1}{2}}+\delta_{4} \delta_{6}^{\frac{1}{2}}\right) .
\end{aligned}
$$

From this fact and (3.20) we have $T_{12} \leq \frac{C}{1+z}\left(\delta_{3}+\delta_{4}^{\frac{1}{2}} \delta_{6}^{\frac{1}{2}}+\delta_{4} \delta_{6}^{\frac{1}{2}}\right)$.

By (3.5), (3.17), and (3.19),

$$
\begin{aligned}
T_{13} & \leq C n E \mathbb{I}\left(\delta \geq \frac{z}{4}\right)(\widetilde{Y}(\rho)-\widehat{Y}(\rho))^{2} \\
& \leq \frac{C n}{z}\left\{E \delta^{3}\right\}^{\frac{1}{3}}\left\{E|\widetilde{Y}(\rho)-\widehat{Y}(\rho)|^{3}\right\}^{\frac{2}{3}} \\
& \leq \frac{C \delta_{3}}{1+z} .
\end{aligned}
$$

Hence, $T_{12}+T_{13} \leq \frac{C}{1+z}\left(\delta_{3}+\delta_{4}^{\frac{1}{2}} \delta_{6}^{\frac{1}{2}}+\delta_{4} \delta_{6}^{\frac{1}{2}}\right)$.

Step 3. $\left|T_{2}\right| \leq \frac{C}{(1+z)}\left(\frac{\delta_{4}^{\frac{1}{2}}}{n^{\frac{1}{4}}}+\delta_{4}\right)$.

We will bound $T_{2}$ by using the technique from Lemma 9 and Lemma 10 of Ho and Chen(Ho, S.T., 1978). Let

$$
\begin{aligned}
G= & \widehat{Y}_{z}\left(I, M_{1}, \ldots, M_{d-1}\right)+\widehat{Y}_{z}\left(K, L_{1}, \ldots, L_{d-1}\right) \\
& -\widehat{Y}_{z}\left(I, L_{1}, \ldots, L_{d-1}\right)-\widehat{Y}_{z}\left(K, M_{1}, \ldots, M_{d-1}\right)
\end{aligned}
$$

and

$$
A=\left\{\tau_{i}(I) \neq L_{i}, \tau_{i}(K) \neq M_{i}, \tau_{i}(I) \neq M_{i}, \tau_{i}(K) \neq L_{i} ; 1 \leq i \leq d-1\right\}
$$

By the same argument as Neammanee and Rattanawong(Neammanee, K., 2009),

$$
\left|T_{2}\right| \leq n\left|E g_{z}^{\prime}(\widehat{Y}(\tau))\left[E^{\tau_{1}, \ldots, \tau_{d-1}} G^{2} \mathbb{I}\left(A^{c}\right)-G^{2} \mathbb{I}\left(A^{c}\right)\right]\right|
$$

Let $\mathcal{B}$ be the $\sigma$-algebra generated by

$$
\left\{I, K, L_{1}, \ldots, L_{d-1}, M_{1}, \ldots, M_{d-1}, Y\left(i_{1}, \ldots, i_{d}\right): 1 \leq i_{1}, \ldots, i_{d} \leq n\right\}
$$

By the fact that $E^{\mathcal{B}} \mathbb{I}\left(A^{c}\right) \leq \frac{C}{n}$ (Neammanee, K., 2008) we have

$$
E|G|^{k} \mathbb{I}\left(A^{c}\right)=E|G|^{k} E^{\mathcal{B}} \mathbb{I}\left(A^{c}\right)=\frac{C}{n} E|G|^{k}=\frac{C \delta_{k}}{n^{\frac{k+1}{2}}} \text { for } k \in \mathbb{N} .
$$

We observe that for $w \leq \frac{z}{2}$,

$$
\begin{aligned}
\left|g_{z}^{\prime}(w)\right| & =\left|[1-\Phi(z)]\left[1+\sqrt{2 \pi} w e^{\frac{1}{2} w^{2}} \Phi(w)\right]\right| \\
& \leq\left\{\frac{e^{-\frac{z^{2}}{2}}}{z}\right\}\left[1+\sqrt{2 \pi}\left(\frac{z}{2}\right) e^{\frac{z^{2}}{8}} \Phi\left(\frac{z}{2}\right)\right] \\
& \leq \frac{\sqrt{2 \pi} e^{-\frac{3 z^{2}}{8}}}{2}+\frac{e^{-\frac{z^{2}}{2}}}{z} \\
& \leq \frac{C}{1+z}
\end{aligned}
$$

where we have used the fact that $1-\Phi(z) \leq \frac{e^{\frac{z^{2}}{2}}}{z}$ for $z>0$ (Barbour, A.D., 2005) in the second inequality. 
From this fact, (2.5), (3.6), (3.21), (3.22), we have

$$
\begin{aligned}
\left|T_{2}\right| \leq & n\left|E g_{z}^{\prime}(\widehat{Y}(\tau)) \mathbb{I}\left(\widehat{Y}(\tau) \leq \frac{z}{2}\right)\left[E^{\tau_{1}, \tau_{2}, \cdots, \tau_{d-1}} G^{2} \mathbb{I}\left(A^{c}\right)-G^{2} \mathbb{I}\left(A^{c}\right)\right]\right| \\
& +n\left|E g_{z}^{\prime}(\widehat{Y}(\tau)) \mathbb{I}\left(\widehat{Y}(\tau)>\frac{z}{2}\right)\left[E^{\tau_{1}, \tau_{2}, \cdots, \tau_{d-1}} G^{2} \mathbb{I}\left(A^{c}\right)-G^{2} \mathbb{I}\left(A^{c}\right)\right]\right| \\
\leq & \frac{C n}{1+z} E G^{2} \mathbb{I}\left(A^{c}\right)+C n\left|E \mathbb{I}\left(\widehat{Y}(\tau)>\frac{z}{2}\right)\left[E^{\tau_{1}, \tau_{2}, \cdots, \tau_{d-1}} G^{2} \mathbb{I}\left(A^{c}\right)-G^{2} \mathbb{I}\left(A^{c}\right)\right]\right| \\
\leq & \frac{C \delta_{2}}{(1+z) \sqrt{n}}+C n\left(E \mathbb{I}\left(\widehat{Y}(\tau)>\frac{z}{2}\right) E\left(E^{\tau_{1}, \tau_{2}, \cdots, \tau_{d-1}} G^{2} \mathbb{I}\left(A^{c}\right)-G^{2} \mathbb{I}\left(A^{c}\right)\right)^{2}\right)^{\frac{1}{2}} \\
\leq & \frac{C \delta_{2}}{(1+z) \sqrt{n}}+\frac{C n}{(1+z)}\left(E \widehat{Y}^{2}(\tau)\left(E G^{2} \mathbb{I}\left(A^{c}\right) E G^{2}+E G^{4} \mathbb{I}\left(A^{c}\right)\right)\right)^{\frac{1}{2}} \\
\leq & \frac{C \delta_{2}}{(1+z) \sqrt{n}}+\frac{C n}{(1+z)}\left(\left(1+\sqrt{n} \delta_{4}\right)\left(\frac{\delta_{4}}{n^{2} \sqrt{n}}\right)\right)^{\frac{1}{2}} \\
\leq & \frac{C}{(1+z)}\left(\frac{\delta_{4}^{\frac{1}{2}}}{n^{\frac{1}{4}}}+\delta_{4}\right) .
\end{aligned}
$$

Step 4. $\left|T_{3}\right| \leq \frac{C}{(1+z)}\left(\frac{\delta_{4}^{\frac{1}{2}}}{n^{\frac{1}{4}}}+\sqrt{n} \delta_{4}^{2}\right)$.

By the same argument as Chen and Shao(Chen, L.H.Y., 2001), we can show that $E\left|g_{z}^{\prime}(\widehat{Y}(\tau))\right| \leq \frac{C}{(1+z)^{2}}\left(1+\sqrt{n} \delta_{4}\right)$, for $z>0$. From this fact and Lemma 2.3, we have

$$
\begin{aligned}
\left|T_{3}\right| & =\left|E g_{z}^{\prime}(\widehat{Y}(\tau))-E g_{z}^{\prime}(\widehat{Y}(\tau)) E \int_{-\infty}^{\infty} K(t) d t\right| \\
& =\left|E g_{z}^{\prime}(\widehat{Y}(\tau))\right|\left|1-E \int_{-\infty}^{\infty} K(t) d t\right| \\
& \leq \frac{C\left(1+\sqrt{n} \delta_{4}\right)}{(1+z)^{2}}\left|1-\frac{n-1}{4} E(\widetilde{Y}(\rho)-\widehat{Y}(\rho))^{2}\right| \\
& \leq \frac{C}{(1+z)^{2}}\left(1+\sqrt{n} \delta_{4}\right)\left(\frac{\delta_{4}^{\frac{1}{2}}}{n^{\frac{1}{4}}}+\delta_{4}\right) \\
& \leq \frac{C}{(1+z)}\left(\frac{\delta_{4}^{\frac{1}{2}}}{n^{\frac{1}{4}}}+\sqrt{n} \delta_{4}^{2}\right) .
\end{aligned}
$$

From (3.11) and step 1 - step 4, we have the theorem.

Remark There are various alternative ways to select the points $X_{i}^{\prime}$ s for estimation of integrals over multidimensional domains. For examples, simple random sampling, lattice sampling( Patterson, H.D., 1954), the orthogonal arrays((Laipaporn, K., 2007), (Loh, W.L., 1996a), (Owen, A.B., 1992) and (Tang, B., 1993)), scrambled net((Owen, A.B., 1997a) and (Owen, A.B., 1997b)).

\section{Acknowledgements}

The authors would like to thank the referees for many helpful remarks which have led us to improve the presentation and the Thailand Research Fund for financial support.

\section{References}

Barbour, A.D. \& Chen, L.H.Y. (2005). An introduction to Stein's method. Singapore: Singapore University Press.

Chen, L.H.Y. \& Shao, Q.M. (2001). A non-uniform Berry-Esseen bound via Stein's method. Probab. Theory Relat. Fields., 120, 236-254.

Ho, S.T. \& Chen L.H.Y. (1978). An $L_{p}$ bound for the remainder in a combinatorial central limit theorem. Ann. Probab., 6, 231-249.

Laipaporn, K. \& Neammanee, K. (2007). A non-uniform bound on normal approximation of randomized orthogonal array sampling designs. International Mathematical Forum, 48, 2347-2367. 
Loh, W.L. (1996a). A combinatorial central limit theorem for randomized orthogonal array sampling designs. Ann Statist., 24, 1209-1224.

Loh, W.L. (1996b). On Latin hypercube sampling. Ann. Stist., 24, 2058-2080.

McKay, M.D., Conover, W.J. \& Beckman, R.J. (1979). A comparison of three methods for selecting values of input variables in the analysis of output from a computer code. Technometrics, 21, 239-245.

Neammanee, K. \& Rattanawong, P. (2009). A constant on a uniform bound in normal approximation for Latin hypercube sampling, to be appear.

Neammanee, K. \& Rattanawong, P. (2008). A uniform bound on the generalization of a combinatorial central limit theorem. International Mathematical Forum, 3, 11-27.

Neammanee, K. \& Suntornchost, J. (2005). A uniform bound on a combinatorial central limit theorem. Stochastic Analysis and Applications., 23, 559-578.

Owen, A.B. (1997a). Monte-Carlo variance of scrambled net quadrature. SIAM J. Numer. Anal., 34, 1884-1910.

Owen, A.B. (1992). Orthogonal array for computer experiments, integration and visualization. Statist. Sinica, 2, 439-452.

Owen, A.B. (1997b). Scrambled net variance for integrals of smooth functions. Ann. Statist., 25, 1541-1562.

Patterson, H.D. (1954). The errors of lattice sampling. J.R. Statist. Soc. Ser.B, 16, 140-149.

Stein, C.M. (1972). A bound for the error in the normal approximation to the distribution of a sum of dependent randon variables., Proc. Sixth Berkeley Symp. mMath. Statist. Probab., 2, 583-602. Univ. California Press, Berkeley.

Stein, C.M. (1986). Approximate computation of expectations. California:IMS, Hayward.

Stein, M.L. (1987). Large sample properties of simulations using Latin hypercube sampling. Technometrics, 29, 143-151.

Tang, B. (1993). Orthogonal array-based Latin hypercubes. J. Amer. Statist. Assoc., 88, 1392-1397. 